\title{
Design Optimization and Implementation of Genetic-Based Moving Sliding Manifold Strategy for Parallel Operation of DC-AC Converters
}

\author{
En-Chih Chang, Hairong Wang, Kuo-Yuan Liao, and Rong-Ching Wu
}

\begin{abstract}
In this paper, the optimal design to parallel operation of DC-AC converters using genetic-based moving sliding manifold strategy is proposed. The AC output voltage regulation and balanced current-sharing among the parallel modules are achieved by the use of the moving sliding manifold (MSS). However, while the load condition of the parallel-connected DC-AC converter is a large parameter variation, the chatter around the MSS occurs. The chatter may cause heat losses and high voltage harmonics in parallel-connected DC-AC converter output, and thus deteriorates system stability and reliability. To eliminate the chatter, the control gains of the MSS can be optimally adjusted by the use of the genetic algorithm (GA). With the proposed strategy, the parallel operation of the DC-AC converter yields a high-quality AC output voltage with low voltage harmonics and fast dynamic response even under large parameter variations, thus achieving more robust system. Experimental results are performed to demonstrate the proposed strategy.
\end{abstract}

Index Terms-Moving sliding manifold (MSS), chatter, genetic algorithm (GA), parallel operation, DC-AC converter.

\section{INTRODUCTION}

Owing to the design ease in thermal management and the remarkable improvement in redundancy, modularity, and maintainability, the parallel operation of DC-AC converters is popularly used in telecommunication electronic systems, like computers, LANs, Modems, Hubs, etc. The parallel operation of the DC-AC converter must supply high-quality AC output voltage with low total harmonic distortion (THD), fast dynamic response, and zero steady-state errors; these requirements can be obtained by using feedback control technologies. To provide the suitable parallel operation of DC-AC converters, a voltage control loop and a current control loop are indispensable. The voltage control loop is designed to obtain the output voltage at the desired amplitude and frequency. The current control loop is designed to control the current-sharing among the parallel modules. Generally, a proportional integral (PI) controller can be used to meet the above requirements of converter design. However,

Manuscript received May 10, 2015; revised August 29, 2015. This work was supported by the Ministry of Science and Technology of Taiwan, R.O.C., under contract number MOST103-2221-E-214-027.

En-Chih Chang, Kuo-Yuan Liao, and Rong-Ching Wu are with the Department of Electrical Engineering, I-Shou University, Kaohsiung City 84001, Taiwan, R.O.C. (e-mail: enchihchang@isu.edu.tw, isu10201017M@cloud.isu.edu.tw, rcwu@isu.edu.tw).

Hairong Wang is with the University of Electronic Science and Technology of China, Chengdu 611731, P.R.C. (e-mail: juliawhr@hotmail.com). the performance of the converter with the PI controller is disappointing under rectified load conditions [1], [2]. Advanced nonlinear control theory should be adopted to ensure good performance. Recently, deadbeat control, $\mathrm{H}$-infinity control, and repetitive control have been proposed for the design of DC-AC converters in parallel operation. However, these control methods can fulfill only one of the performance requirements [3]-[5]. Some literatures have indicated that the sliding mode control (SMC) strategy deserves special attention, because this method provides a systematic approach to maintain asymptotic stability and consistent performance [6], [7]. A numerous publications of the SMC have also been applied to the control of DC-AC converters in parallel operation [8]-[13]. Unfortunately the fixed switching manifolds are employed. These switching manifolds are determined independently of the initial states. The SMC with these fixed switching manifolds are sensitive to parameter variations and external disturbances in the reaching phase. Thus, in this paper, a MSS adaptable to arbitrary initial conditions is proposed to remove the drawback. Such manifold is initially designed to pass the initial conditions and is subsequently moved towards a predetermined sliding manifold by rotating or/and shifting [14]-[16]. By the use of the MSS, the system sensitivity to uncertainties is reduced through accelerating the reaching phase. Because the MSS always passes through the vicinity of the representative point of the system, the system robustness can be improved. However, once a large parameter variation is applied, the MSS controlled system has a chatter problem, thus resulting in serious output-voltage harmonics in the parallel operation of the DC-AC converter. Proposed in 1975, genetic algorithm (GA) is a stochastic search technique that guides the principles of evolution and natural selection in a population towards an optimum using genetics [17]-[19]. Hence, the control gains of the MSS can be optimally determined by GA, and then the chatter can be eliminated. Finally, the proposed strategy is demonstrated by the DC-AC converter in parallel operation, and is implemented digitally by using a DSP. Experimental results show the feasibility and advantages of using the proposed strategy.

\section{Dynamic Model of DC-AC ConVerter IN PARALLEL OPERATION}

Fig. 1 shows the parallel operation of DC-AC converter, and Fig. 2 is its equivalent circuit. From Fig. 2, the converter dynamics can be expressed as 


$$
\left\{\begin{array}{c}
u_{1}=R_{1} i_{L_{1}}+L_{1} \frac{d i_{L_{1}}}{d t}+v_{O} \\
\cdot \\
\cdot \\
\cdot \\
u_{n}=R_{N 1} i_{L_{N}}+L_{N} \frac{d i_{L_{N 1}}}{d t}+v_{O} \\
i_{L_{1}}+i_{L_{2}}+\cdots+i_{L_{N}}=i_{C_{1}}+i_{O} \\
i_{C_{1}}=C_{T} \frac{d v_{O}}{d t}
\end{array}\right.
$$

where $C_{T}=\sum_{i=1}^{N} C_{i}$.

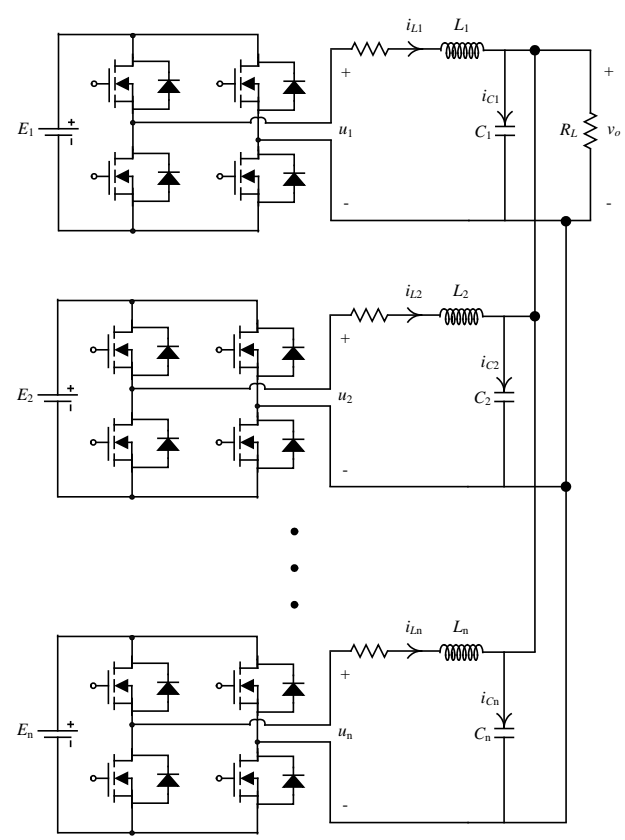

Fig. 1. Circuit diagram of DC-AC converter in parallel operation.

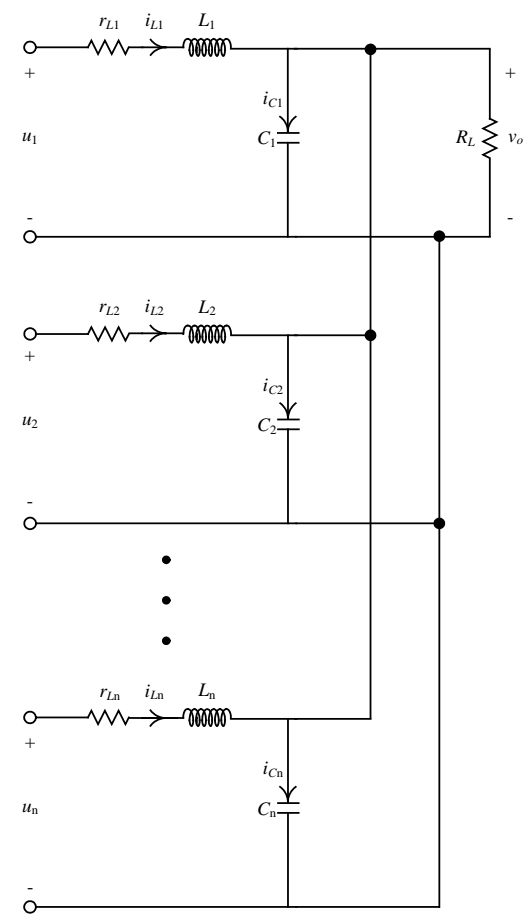

Fig. 2. Equivalent circuit of DC-AC converter in parallel operation.
Firstly, a state variable $e_{1}$ related to the state error between the output voltage and the reference voltage is defined as

$$
e_{1}=v_{O}-v_{r e f}
$$

where $v_{O}$ is the output voltage, and $v_{r e f}=V_{m} \sin (\omega t)$ is the reference voltage.

Our aim is to design a control law, $u_{p}=u_{e}+u_{s}$ completely, thus yielding output voltage regulation and balanced current-sharing among the parallel modules. The control law is derived as follows.

\section{CONTROL Design}

\section{A. Moving Sliding Manifold (MSS)}

Define the state vectors as

$$
\left\{\begin{array}{l}
x_{p}=\left[\begin{array}{ll}
x_{p 1} & x_{p 2}
\end{array}\right]^{T}=\left[\begin{array}{ll}
v_{o} & \dot{v}_{o}
\end{array}\right]^{T} \\
x_{m}=\left[\begin{array}{ll}
x_{m 1} & x_{m 2}
\end{array}\right]^{T}=\left[\begin{array}{ll}
v_{\text {ref }} & \dot{v}_{\text {ref }}
\end{array}\right]^{T} \\
u_{p}=u_{i} \\
u_{m}=u_{\text {ref }}
\end{array}\right.
$$

The dynamics of the plant given by (3) can be restated as

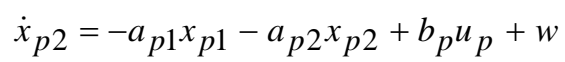

where $a_{p 1}=\frac{1}{L C}, a_{p 2}=0, b_{P}=\frac{K_{P W M}}{L C}, w=-\frac{1}{C} \frac{d}{d t} i_{O}$ is the disturbance, and $u_{p}$ is the plant input.

The dynamics of the reference model in (3) can be restated as

$$
\dot{x}_{m 2}=-a_{m 1} x_{m 1}-a_{m 2} x_{m 2}+b_{m} u_{m}
$$

where $a_{m 1}=\omega_{0}^{2}, a_{m 2}=0, b_{m}=1, u_{m}$ is the reference input.

Subtract (4) from (5), and let $e_{1}=x_{m 1}-x_{p 1}$ : the error differential equations are

$$
\begin{gathered}
\dot{e}_{1}=e_{2}=\dot{x}_{m 1}-\dot{x}_{p 1}=x_{m 2}-x_{p 2} \\
\dot{e}_{2}=-a_{m 1} e_{1}-a_{m 2} e_{2}+\left(a_{p 1}-a_{m 1}\right) x_{p 1} \\
+\left(a_{p 2}-a_{m 2}\right) x_{p 2}+b_{m} u_{m}-b_{p} u_{p}-w
\end{gathered}
$$

The derivatives may be approximated by Euler's method

$$
\dot{e}(n) \cong \frac{e(n+1)-e(n)}{T}
$$

where $T$ is the sample interval in seconds.

This approximation can be used in place of all derivatives that appear in the controller differential equations (6) and (7), 
to yield a set of equations that can be solved using a digital computer:

$$
\begin{gathered}
e_{1}(n+1)=e_{1}(n)+T e_{2}(n) \\
e_{2}(n+1)=e_{2}(n)+T \dot{e}_{2}(n) \\
=e_{2}(n)+T\left[-\sum_{i=1}^{2} a_{m i} e_{i}(n)\right. \\
+\sum_{i=1}^{2}\left(a_{p i}-a_{m i}\right) x_{p i}+b_{m} u_{m}-b_{p} u_{p}-w
\end{gathered}
$$

Then, the moving sliding manifold is selected as

$$
\sigma(n)=c_{1}(n) e_{1}(n)+e_{2}(n)-\alpha(n)
$$

From (11), $\sigma(n+1)$ is

$$
\sigma(n+1)=c_{1}(n+1) e_{1}(n+1)+e_{2}(n+1)-\alpha(n+1)
$$

Consider the discrete reaching law as

$$
\sigma(n+1)=(1-q T) \sigma(n)-\varepsilon T \operatorname{sgn} \sigma(n)
$$

Further define

$$
\varepsilon=p_{1}|\sigma(n)|
$$

where $p_{1}$ is a constant.

Then (13) can be written as

$$
\begin{aligned}
\sigma(n+1) & =(1-q T) \sigma(n)-\varepsilon T \operatorname{sgn} \sigma(n) \\
& =\left(1-q T-p_{1} T\right) \sigma(n) \\
& =q_{1} \sigma(n)
\end{aligned}
$$

where $q_{1}=1-q T-p_{1} T,-1<q_{1}<1$.

Introduce the control function

$$
u_{p}=u_{e}+u_{s}
$$

In the following, the equivalent control law $u_{e}$, which will determine the dynamics of the system on the sliding manifold, is derived. The equivalent control law is derived as

$$
\sigma(n+1)=q_{1} \sigma(n)
$$

Assume that $a_{p_{i}}=\bar{a}_{p i}+\Delta a_{p i}, i=1,2 \quad$ and $b_{p}=\bar{b}_{p}+\Delta b_{p}$.

The '-' symbol represents the system parameter in the nominal case, which is defined as the case I which the plant model is known exactly without external disturbance.

Substituting $u_{p}=u_{e}, a_{p_{i}}=\bar{a}_{p i}, b_{p}=\bar{b}_{p}$ into yields

$$
\begin{aligned}
& -q_{1}\left[c_{1}(n) e_{1}(n)+e_{2}(n)-\alpha(n)\right]+c_{1}(n+1) e_{1}(n) \\
& +T c_{1}(n+1) e_{2}(n)+e_{2}(n) \\
& +T\left[-\sum_{i=1}^{2} a_{m i} e_{i}(n)+\sum_{i=1}^{2}\left(\bar{a}_{p i}-a_{m i}\right) x_{p i}+b_{m} u_{m}\right] \\
u_{e}= & \frac{-\alpha(n+1)}{T \bar{b}_{p}}
\end{aligned}
$$

However, if the system parameters are perturbed or unknown, then the equivalent control law design does not guarantee the performance described by (18). Moreover, the stability of the controlled system may be destroyed.

The function $u_{s}$ is constructed as (19) to ensure the system performance given by (16), despite uncertain system dynamics.

$$
u_{s}=\varphi_{1} e_{1}+\varphi_{2} e_{2}+\varphi_{3} x_{p 1}+\varphi_{4} x_{p 2}+\varphi_{5} u_{m}+\varphi_{d}
$$

Therefore the problem of controller design is to yield the control law $u_{p}$ such that the sliding condition (21) is satisfied.

$$
[\sigma(n+1)-\sigma(n)] \sigma(n)<0
$$

Substituting (11) and (12) into (20), then sufficient conditions for stability is guaranteed.

Notably that the control law (20) implies the signum function cross the manifold $\sigma(n)$. Hence, chatter occurs. A solution to this problem is to smooth the discontinuous control law in a thin boundary layer. The discontinuous function can be replaced by the following continuous function:

$$
\frac{\sigma(n)}{|\sigma(n)|+\eta}
$$

where $\eta$ is positive constant.

Notice that though the (16) provides the insensitivity to system uncertainties through accelerating the reaching phase, the chatter still occurs once a large parametric variation is applied. Thus, an optimal solution for eliminating the chatter is employed by the use of the GA.

\section{B. Genetic Algorithm-Based Tuning}

The real-coded GA (RGA) is employed to tune the control gains of the MSS. Compared to binary GA, the RGA is unnecessary to transform the real numbers into binary numbers, and thus has the superiority of speed and precision. The flow chart of RGA is depicted in Fig. 3.

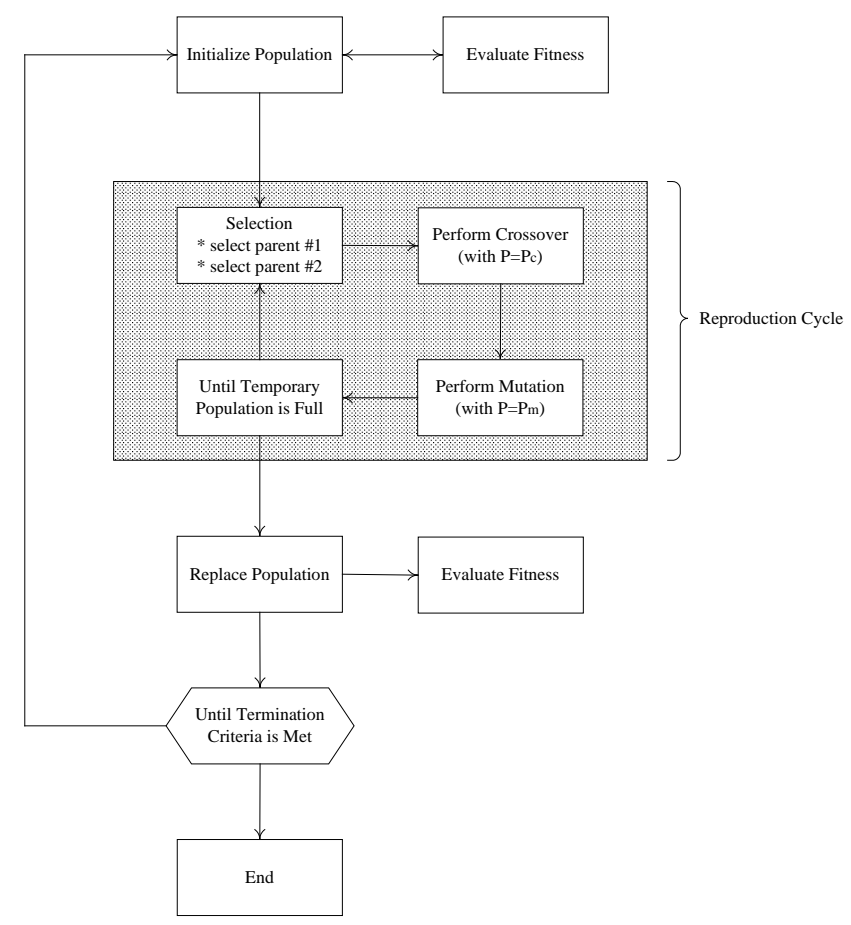

Fig. 3. Flow chart of RGA. 


\section{1) Initial population}

An initial population is constructed as

$$
x(n)=x_{\min }(n)+\operatorname{rand} \times\left(x_{\max }(n)-x_{\min }(n)\right), n=1,2, \ldots, j
$$

where rand is bounded by 0 and 1 , and $x_{\min }(n)$ and $x_{\max }(n)$ are minimum and maximum values, respectively.

\section{2) Fitness function}

The objective function, $J$ is designed as

$$
J=\int_{0}^{\infty}\left(W_{1}\left|x_{1}\right|+W_{2} u^{2}\right) d t
$$

where $W_{1}$ and $W_{2}$ are both weight values.

Then, the fitness function, $F$ is selected as

$$
F=\frac{1}{J}
$$

\section{3) Selection}

By simulating the spinning of a suitably weighted roulette wheels, fitness proportionate selection can be performed. Suppose the generation size be $g$, the adaptive degree of individual $k$ be $F(k)$, the proportion of individual $k$ can be obtained as

$$
P(k)=\frac{F(k)}{\sum_{k=1}^{g} F(k)}, \quad k=1,2, \ldots, g
$$

\section{4) Crossover}

Choose a crossover site $K_{c r}$ within $0 \leq K_{c r}<M$ randomly, then by keeping the genes of the parent creatures between position 1 and $K_{c r}$ unaltered and interchanging the genes of the parent creatures between position $K_{c r}+1$ and $M$, two new creatures yields

$$
\begin{gathered}
x^{c r, r, d}=\alpha x^{s, r, d}(n)+(1-\alpha) x^{s, r, h}(n) \\
x^{c r, r, h}=(1-\alpha) x^{s, r, d}(n)+\alpha x^{s, r, h}(n)
\end{gathered}
$$

where $n$ is bounded by $K_{c r}+1$ and $M$, and $0 \leq \alpha \leq 1$.

\section{5) Mutation}

The $(r+1)$ th population can be created by using the mutation operation as follows.

$$
x^{r+1, d}(n)=x^{c r, r, d}(n)+\Theta \zeta_{n}
$$

where $\Theta$ is bounded by -1 and 1 , and $\zeta_{n}$ is the mutation amplitude of the $n$th gene.

\section{EXPERIMENTAL RESULTS}

Using proposed theoretical analysis and the dynamic model of the DC-AC converter in parallel operation, then the parallel operation of the DC-AC converter is designed with system parameters given as follows: $E_{1}=E_{2}=E_{3}=210 \mathrm{~V}$, $L_{1} \cong L_{2} \cong L_{3} \cong 0.2 \mathrm{mH} \quad, \quad C_{1}=30 \mu \mathrm{F} \quad$, $r_{1} \cong r_{2} \cong r_{3} \cong 0.05 \Omega, R_{L}=12 \Omega$, output voltage, $v_{o}=110$
$V_{r m s}, 60 \mathrm{~Hz}$. The majority of sensitive loads are rectifier loads. When the diodes are conducting, the parallel operation of the DC-AC converter is exposed to a large filter capacitor, and when they are not conducting, the parallel operation of the DC-AC converter is practically in no load condition. Therefore, the proposed strategy must be able to correctly regulate output voltage with minimum distortion. Fig. 4(a) shows both the output voltage and the load current with the proposed strategy when parallel operation of the DC-AC converter is loaded with a full-wave rectifier followed by a $100 \mu \mathrm{F}$ capacitor in parallel with a $35 \Omega$ resistor; the \%THD is approximately $2.04 \%$, which can be regarded as a good performance in the parallel operation of the DC-AC converter. However, Fig. 4(b) with the classic SMC under the same test condition exhibits a high voltage \%THD of 9.31\%. The values of filter parameters ( $L$ and $C$ ) are supposed in suffering from $20 \% \sim 400 \%$ of nominal values while the solar inverter system is under $12 \Omega$ resistive load. Fig. 4(c) and Fig. 4(d) display the experimental output voltage waveforms of the solar inverter controlled by the proposed strategy and the classic SMC. The proposed strategy is insensitive to the parameter variations than the classic SMC. Indeed, the proposed strategy is capable of maintaining a good steady-state and dynamic response, and shows significant improvement in reducing the THD of the output voltage even under large parameter variations. The output voltage exhibits a low \%THD of $1.31 \%$, with the proposed strategy and a high \%THD of $10.35 \%$ with the classic SMC.

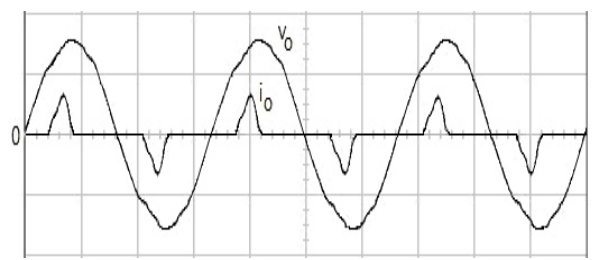

(a) $(100 \mathrm{~V} /$ div; 30A/div; 5ms/div)

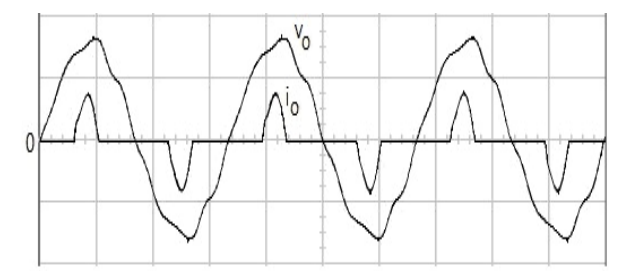

(b) $(100 \mathrm{~V} / \mathrm{div} ; 30 \mathrm{~A} / \mathrm{div} ; 5 \mathrm{~ms} / \mathrm{div})$

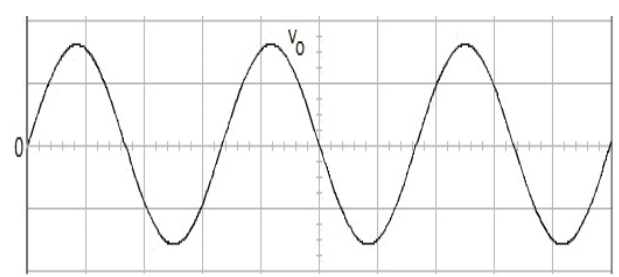

(c) $(100 \mathrm{~V} /$ div; $5 \mathrm{~ms} /$ div $)$

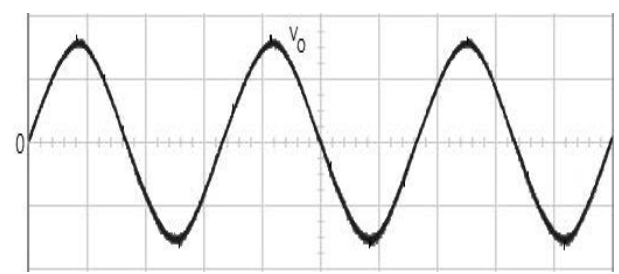

(d) $(100 \mathrm{~V} /$ div; $5 \mathrm{~ms} /$ div $)$

Fig. 4. Experimental waveforms under (a) Rectifier load with the proposed strategy. (b) Rectifier load with the classic SMC. (c) LC parameter variation with the proposed strategy. (d) LC parameter variation with the classic SMC. 


\section{CONCLUSIONS}

For more precise tracking control, this paper presents a GA-based MSS strategy for the parallel operation of the DC-AC converter so that the high-quality AC output voltage and the current-sharing among the parallel modules are obtained. The MSS has superior robustness compared to the fixed sliding manifold because it ensures the sliding mode occurrence from an arbitrary initial state. By the use of the GA, the chatter around the MSS can be eliminated. The proposed strategy is implemented in a laboratory prototype by means of a DSP. Experimental results show low THD of output voltage, which overmatches with the industrial standard 5\% even under large parameter variations.

\section{ACKNOWLEDGMENT}

This work was supported by the Ministry of Science and Technology of Taiwan, R.O.C., under contract number MOST103-2221-E-214-027.

\section{REFERENCES}

[1] R. Kayalvizhi, S. P. Natarajan, D. Sivakumar, and K. B. Elangovan, "Design and simulation of PI control for paralleled positive output elementary Luo converters for distributed power supplies," in Proc. IEEE Int. Conf. Power India Conference, 2006, pp. 1-5.

[2] B. H. Jassim, B. Zahawi, and D. Atkinson, "Simple control method for parallel connected three-phase PWM converters," in Proc. Int. Conf. Power Electronics, Machines and Drives, 2012, pp. 1-5.

[3] M. Wang, F. Z. Li, Y. D. Liu, L. P. Huang, and M. Sakane, "Distributed parallel operation of modified deadbeat controlled UPS inverters," in Proc. IEEE Int. Conf. Power Electronics Specialists Conference, 2007, pp. $1727-1732$.

[4] T. Hornik and Q. C. Zhong, "Parallel PI voltage-Ho current controller for the neutral point of a three-phase inverter," IEEE Trans. on Industrial Electronics, vol. 60, no. 4, pp. 1335-1343, 2013.

[5] B. Y. Ren, H. Bai, X. D. Sun, Q. I. Zhang, and S. L. An, "Control strategy for wireless paralleled inverters under nonlinear load conditions," in Proc. Int. Conf. Electronics and Application Conference and Exposition, 2014, pp. 1188- 1192.

[6] Y. Shtessel, C. Edwards, L. Fridman, and A. Levant, Sliding Mode Control and Observation, Springer, New York, 2014.

[7] J. K. Liu and X. H. Wang, Advanced Sliding Mode Control for Mechanical Systems Design, Analysis and MATLAB Simulation, Springer-Verlag, Heidelberg, Berlin, 2012.

[8] D. Biel, R. Ramos, F. Guinjoan, J. Negroni, and C. Meza, "Sliding-mode control design of parallel-connected switching converters for modular transformerless DC-AC step-up conversion," in Proc. Int. Symp. Circuits and Systems, 2005, vol. 4, pp. 3067 - 3070.

[9] L. C. Gallegos, N. Vazquez, C. Hernandez, M. Sema, and A. D. Hernandez, "Parallel inverters in photovoltaic systems for isolated applications," in Proc. IEEE Int. Autumn Meeting on Power, Electronics and Computing, 20014, pp. 1-6.

[10] R. Ramos, D. Biel, E. Fossas, and F. Guinjoan, "Interleaving Quasi-Sliding-Mode Control of Parallel-Connected Buck-Based Inverters," IEEE Trans. on Industrial Electronics, vol. 55, no. 11, pp. 3865-3873, 2008.

[11] Y. Zhang and Z. H. Jiang, "Sliding mode based zero-sequence current mitigation of parallel-connected power converters," in Proc. Int. Conf. Electric Machines and Drives, 2009, pp. 1658-1663.

[12] R. Ramos, D. Biel, E. Fossas, and F. Guinjoan, "Sliding-mode control design applied to parallel-connected modular inverters through FPGA-based implementation," IET Control Theory \& Applications, vol. 3, no. 12, pp. 1611-1624, 2009.

[13] R. J. Wai and W. H. Wang, "Grid-connected photovoltaic generation system," IEEE Trans. on Circuits and Systems I: Regular Papers, vol. 55, no. 3, pp. 953-964, 2008.

[14] S. Tokat, I. Eksin, and M. Guzelkaya, "Linear time-varying sliding surface design based on co-ordinate transformation for high-order systems," Transactions of the Institute of Measurement and Control, vol. 31, no. 1, pp. 51-70, 2009.

[15] L. Li, Q. Z. Zhang, and N. Rasol, "Time-varying sliding mode adaptive control for rotary drilling system," Journal of Computers, vol. 6, no 3, pp. 564-570, 2011

[16] J. Geng, Y. Z. Sheng, and X. D. Liu, "Second-order time-varying sliding mode control for reentry vehicle," International Journal of Intelligent Computing and Cybernetics, vol. 6, no. 3, pp. 272-295, 2013.

[17] J. H. Holland, Adaptation in Natural and Artificial Systems, Michigan: The University of Michigan Press, Ann Arbor, 1975.

[18] H. M. Hasanien and S. M. Muyeen, "Design optimization of controller parameters used in variable speed wind energy conversion system by genetic algorithms," IEEE Trans. on Sustainable Energy, vol. 3, no. 2, pp. 200-208, 2012

[19] S. Abdel-Khalik, G. S. Mostafa, M. I. Masoud, and B. W. Wiliams, "Optimum flux distribution with harmonic injection for a multiphase induction machine using genetic algorithms," IEEE Trans. on Energy Conversion, vol. 26, no. 2, pp. 501-512, 2011.

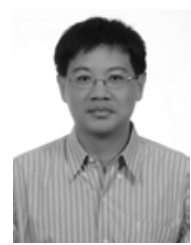

En-Chih Chang was born in Kaohsiung, Taiwan, in 1975. He received the B.S. degree from Feng-Chia University, Taichung, Taiwan, R.O.C., in 1999, the M.S degree from National Taiwan Ocean University, Keelung, Taiwan, R.O.C., in 2001, and the Ph.D. degree from National Cheng Kung University, Tainan, Taiwan, R.O.C., in 2008, all in electrical engineering. He joined the Department of electrical engineering, i-shou university in 2009 as an assistant professor. His research interests include sliding mode control, intelligent control, grey theory, and their applications in power electronics systems.

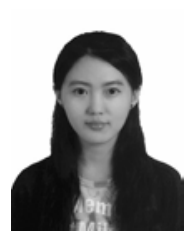

Hairong Wang was born in 1995. She is working toward the B.S. degree in automation engineering from University of Electronic Science and Technology of China. She is currently an exchange student in Department of Mechanical and Automation Engineering from I-Shou University, Kaohsiung City, Taiwan, R.O.C. Her further research interests include sliding mode control, genetic algorithm, machine learning, and neural network.

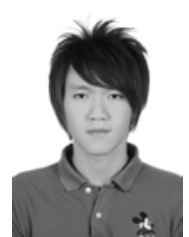

Kuo-Yuan Liao received B.S. degree in electronic engineering from I-Shou University, Dashu District, Kaohsiung City, Taiwan, R.O.C., in 2013, where he is currently working toward the M.S. degree in the Department of Electrical Engineering. His research interests include the field of power electronics.

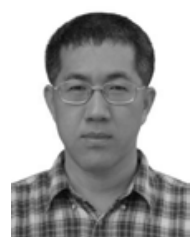

Rong-Ching Wu graduated from National Taiwan Institute of Technology, Taipei, Taiwan, in 1990. He received the M.Sc. degree in 1994 and received the Ph.D. degree in 2001 from National Sun Yat-Sen University, Kaohsiung, Taiwan, R.O.C. From 1991 to 2001, he was an electrical engineer in Taiwan Power Company, Kaohsiung, Taiwan, and was involved with design, construction, and operation of power systems. Currently, he is an assistant professor at I-Shou University, Kaohsiung, Taiwan, R.O.C. 